\title{
SYMPTOMATIC PATTERN MATCHING OF COVID-19 WITH PRE-EXISTING DISEASES TO PREDICT REMEDIES
}

\author{
DRISHTI GUPTA ${ }^{1} \&$ DEEPANSHU AHUJA ${ }^{2}$ \\ *I Master of Food science and technology, IK Gujral Punjab Technical University, Kapurthala, India \\ ${ }^{* 2}$ Bachelor of Medical Laboratory Technology, Lovely Professional University, Jalandhar, India
}

\begin{abstract}
Corona viruses are large family of positive strand RNA viruses and enveloped viruses. There have been four genera of coronavirus, human viruses detected that are Alpha Corona virus and Beta coronavirus genera SARS COV-1 and SARS COV-2 (also known as covid-19, Wuhan virus) are a lineage beta corona viruses while MERS-COV a lineage beta coronavirus. MERS and SARS are globally endemic and cause upper respiratory tract infection. The fertility rate of MERS is highly progressive than SARS.COVID-19 is highly contagious and currently pandemic, outbreak of this virus globally is a major concern. In contrast MARS and SARS like COVs can only cause intense but short lived fatal outbreak. Corona viruses mainly found in bat around the world, it can be found in other species including mice, horses, dog cat, camel and human. This respiratory syndrome cause enteric, neurologic disease, hepatic or multi organ failure which can result in death, especially in individuals who are having comorbidities. The mode of transmission is direct or sometimes indirect. Some patient may be symptomatic or sometime asymptomatic and hence no specific treatment for drug exists for corona virus, control measures and preventions from infection are crucial in order to prevent the spread in human immunity.
\end{abstract}

KEYWORDS: MERS-COV, SARS-COV-1. SARS-COV-2, its comparison, Pathogenesis, Diagnosis, Treatment, Prediction

Received: Sep 07, 2020; Accepted: Sep 27, 2020; Published: Dec 02, 2020; Paper Id.: IJMPSOCT20201

\section{INTRODUCTION}

In human history various endemic and pandemic, the infection has been limiting the longer the longevity and quality of life, which is directly associated with number of deaths and increase of mobility all over the world. Transmission of every emergent pathogen whether it is direct or indirect has the potential to produce and create the negative impact of the general Welfare of every community or economy ${ }^{(7)}$. In December 2019, China was majorly responsible for the spread of novel coronavirus. The Coronavirus belongsto midovirales order of the Coronaviridae family. Several studies have described biological, clinical, demographic, radiological or pathological findings and characteristic which are associated with an infected patient of COVID-19. This virus shows crown-like Spikes on its outer surface, $65-125 \mathrm{bp}$, and envelope positively charged single-strand RNA as a nucleus material - 26 to $32 \mathrm{~kb}$ in length ${ }^{(5)}$. The Coronaviruses are those they may cause many symptoms alike to acute respiratory distress syndrome (ARDS) such as pneumonia, common cold infections, breathing difficulty, fever, and lung infection. 10 to $30 \%$ of upper respiratory tract infections globally infected in adults ${ }^{(2,3)}$. COVID-19 infected patients ranging from 20 to $30 \%$ of patients required ventilation and some of near to $10 \%$ died ${ }^{(19)}$. Related to this existence disease are MARS and SARS viruses are the same family, having near to same symptomatic pattern to SARS-COV-2. In June 
2012 Middle East Respiratory Syndrome (MARS) was detected in a patient who died with this virus in Jeddah, Saudi Arabia moreover, the mass infection was emphasized in the central region (31.7\%), Western region (40.8\%), followed by Eastern region $(20 \%)^{(9)}$. In 2003 (Severe acute renal syndrome) more than 8000 patients were infected and result into 774 deaths worldwide it also negatively affect global economic loss due to reduced international travel and domestic demands

(12). The outbreak of novel coronavirus was initially from the seafood market Wuhan city of China which rapidly infected thousands of people worldwide. Some pre domestic mammals could serve as facilitating recombination, mutation events exception of Genetic diversity, and intermediate host ${ }^{(11) .}$ The hospital-based outbreaks and high mortality rate in domestic areas mostly in patients with various comorbidities for instance renal failure, diabetic and cardiovascular disease, evoked intensive discussion, and major Global concern among the media ${ }^{(10)}$. The similarities between all these three types of Coronaviruses fabricate difficulty to discover drugs and treatment for COVID-19.

\section{Epidemiology}

COVID-19 is a zoonotic source of infection that ecologically origin in bats ${ }^{(5)}$ SARS-COV-2 transmission can be through droplets (particle $>5$ to $10 \mu \mathrm{m}$ in diameter) can be through direct contact (within $1 \mathrm{~m}$ ) within someone who has symptoms like coughing and sneezing, through indirect contact like formalities, airborne transmission, (particles more $5 \mu \mathrm{m} /$ diameter) the particles remain in the air for a long period and can be transmitted to another person over distance more than $1 \mathrm{~m}$ and through faecal-oral transmission ${ }^{(12) .}$ The patients of COVID-19 can be asymptomatic and therefore mode of Transmission can remain undefined. Till Feb 29, 2020, there were 83,652 Laboratories positive confirm cases with a $3.4 \%$ mortality rate (open 2791 death) has been reported.

The primary cause of SARS-COV- 2 transmission is unknown in humans. Infection rate of COVID-19 is $\sim 1.7$ and the basic reproduction number $\left(\mathrm{R}^{\circ}\right)$ world is 2.2 . The incubation time is 12 days, recovery rate rises up to $32 \%$ and the rate of fatality is nearly $3.3 \%$. COVID-19 is associated generally with higher viral load in the upper respiratory tract and its secretions, and it may also evolve towards SARI and ARDS (acute respiratory distress syndrome) ${ }^{(7)}$, most probably in elder patients with immunosuppression and comorbidities. This is now evident that there is an important role of health care services in the amplification of SARS-COV-2. Sometimes SARS-COV-2 also acts as "super spreader" and asymptomatic carriage of infection. The epidemiology analysis between SARS-COV-1 and SARS-COV-2 and MERS reveals a similar pattern. Districts hotspots (in India: (1) Red zone (hotspot) - high doubling rate $<4$ days/active cases. (2) Orange zone (non-hotspots) -fewer cases/no case in last 14 days. (3)Green Zone -without confirmed/new cases in the last 21 to 28 days.

\section{Pathogenesis}

COVID-19 spread properly in the first two months of infection throughout China and caused a higher degree of illness. In the starting of infection, the fertility rate of SARS-COV-2 was less than MERS and SARS-COVID-1 ${ }^{(11)}$ because it has reported that it has a high potential rate to infect the human. Various studies suggested that children and most of the individual have been infected with COVs have potentially able to develop an antibody against the infection with some level of the immune response. Presence of prior antibody without exposure of virus individual can able to produce permanent immunity against special strain or even for the strain of other COVs species with cross-immunity but there is no specific and clear evidence of this theory. The lethality of SARS-COV-2 is that it rises continuously with the elderly age.

\section{Pre-existing disease MERS and SARS}

MERS and SARS enter inside the host cell through its receptor called dipeptidyl peptidase 4. A protein is present for 
cleaving the protease present in the envelope of cell which is required for the fusion of genetic material of the virus in the cell. Inside the cytoplasm, transcription and replication occur on the double-membrane vesicle. The seven subgenome mRNAs from the transcription process via an intermediate of negative strand subgenomic RNA. That subgenomic RNA is nested with $3^{\prime}$ and which are further joined to a ladder encoded with $5{ }^{`}$ of genomic structure. The viral encapsulation of RNA is in the $\mathrm{N}$ protein which is transported to the site of assembly which is called as endoplasmic reticulum Golgi intermediates compartment (GIC) the encapsulated viral RNA in the N protein converted into vesicles from buds into vesicles which are arranged with some other proteins like S, M and E proteins. These vesicles arenow transported to the cell surface for further release resulting in infecting other cells ${ }^{(10)}$.

The similarity between the genomic structure of both viruses is with the protein that is involved in the replication of viruses encodes as a $5^{`}$ and structural protein which are encoded by genes at $3^{\circ}$ of the genomic structure ${ }^{(10)} \mathrm{S}$ protein which is a surface envelope spike protein of SARS and MERS having an important role in detecting tissue tropism and the cell, also in the manifestation of the infection. Some proteins are needed for the virus viability and it is dispersed throughout the structural gene and that might interfere with the innate immunity in infected animals and humans are called accessory proteins ${ }^{(4)}$. The difference between SARS-COV-1and MERS-COV is that they both have a different number of accessory proteins. SARS-C0V-1 has eight different accessory proteins and MERS has five different accessory proteins hence, which have a contrasting effect on signalling and induction of Type 1 interferon that could explains how SARSCOV-1 is more resistant to interferon then MERS. The sensitivity of these interferon and their differences has the repercussion on the treatments because to treat patients those are infected with MERS and the SARS-COV-1 Type 1 interferon has been extensively used. Coronaviruses have a high rate of recombination and mutation tendency to cross host species ${ }^{(10)}$. Though, this property was observed by the spread of SARS-COV-1 into the human population from Chinese horseshoe bat during the epidemic 2002 and $2003^{(10)}$.

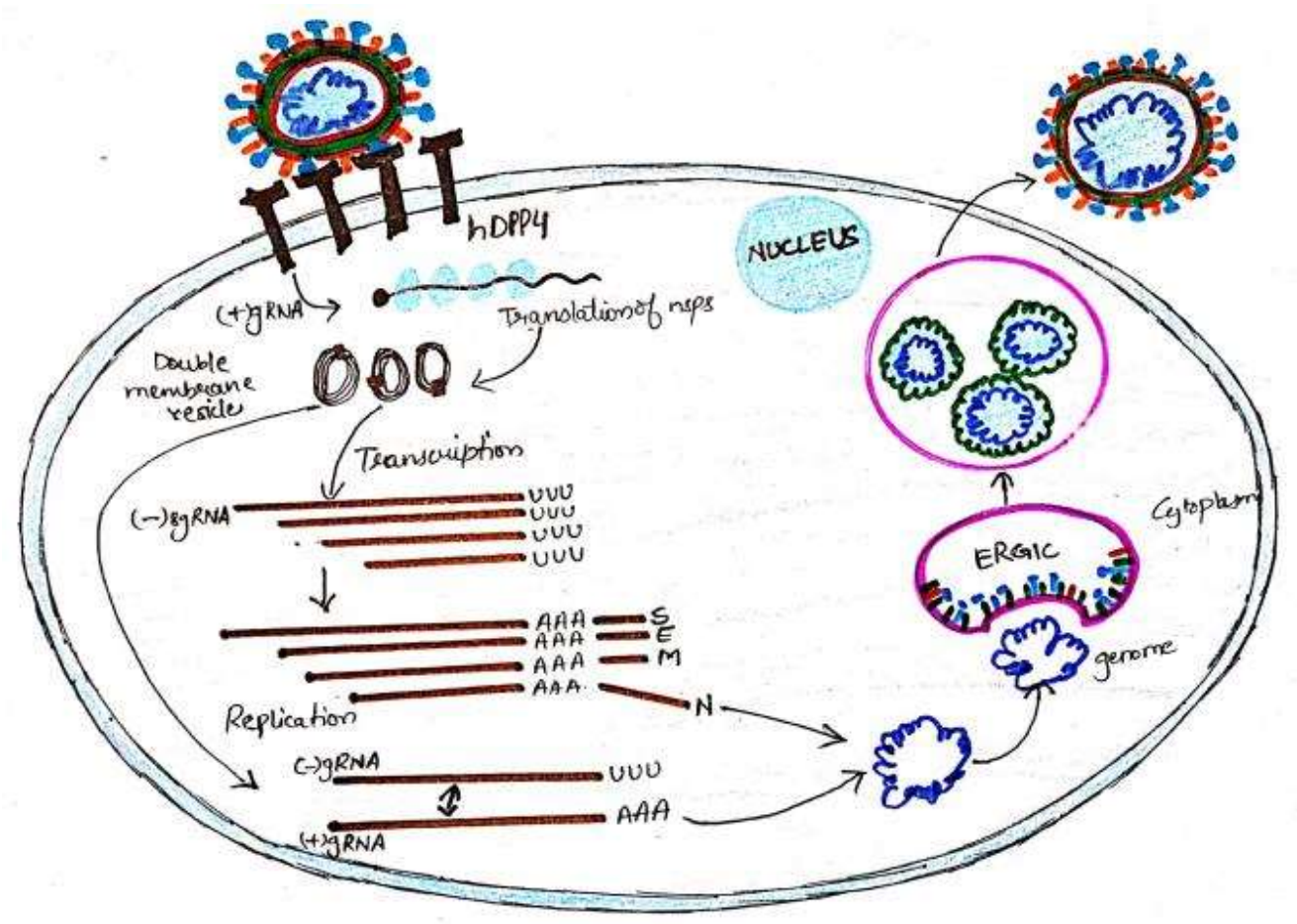

Figure 1: MERS- SARS-COV-1 Life cycle inside the human cell 
MERS-COV-1 is highly concerned because of its ability to adapt to every new changing environment that would increase virulence and enhancing the ability of the transmission amongst individuals, it has been observed. Both MERS and SARS-COV-1binds to an ectopeptidase (DPP4 and angiotensin-converting enzyme) for the entrance inside the host cell without binding to the host cell receptors, it does not show any infection inside the body (10). Although the susceptibility of SARS-COV replication is with the human intestinal cells, the portal of entry through the intestinal tract is still uncertain ${ }^{(4,10)}$. In the body, there is some cell called as colonic enterocytes and hepatocytes where SARS-COV was found, but these cells lacked in ACE2, whereas this virus is also not detected in the blood vessels in its endothelial cell and smooth muscle cells of the intestine despite their testimony of ACE2 also possibly shows the diffuse alveoli damage (DAD) and act as a negative regulator of the local renin-angiotensin system which is called as S protein -ACE2- Reninangiotensin pathway, named because of binding of COVs protein in the body $(2,4,10)$.

Moreover, there are some SARS-COV-encoded protein like 3a and 7a, appear to be strong inducers of apoptosis in cell lines obtained from different organs involve kidney, liver, lungs. DPP4 (also known as CD26) recognized as the functional cellular receptor of MERS-COV ${ }^{(4)}$. SARS-COV originated in bats and further can adapt to known bat variants of ACE2 as cross-species to infect the human population. Then surface glycoprotein can be enhancing the binding with the human receptor ${ }^{(10)}$. It has been discovered that MERS-COV has not mutated subsequently during transmission in human bodies, only a single mutation at P1020 outward protein has been typically detected in humans' inhabitants. In contrast in the role of surface glycoprotein mutation virus's alteration to the new isolates is probably a result of a narrow difference in the process of virus entrance, as its entry includes both the receptor binding and successive release of fusion peptides ${ }^{(10)}$.

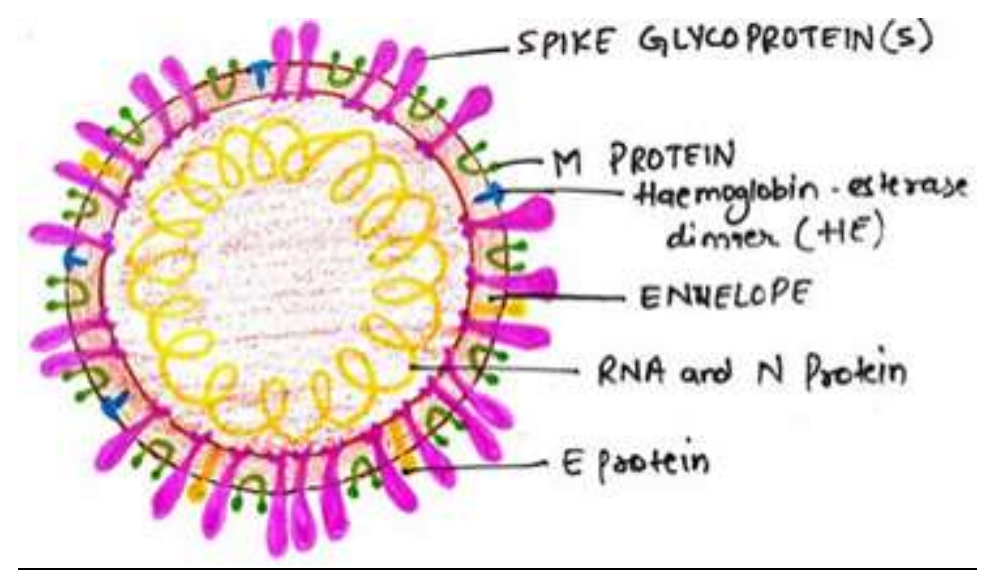

Figure 2: Structure of COVID-19

\section{Covid-19 Pathogenesis}

SARS-COV-2 having spike protein of two domains S1 and S2, S1 is for receptor binding and S2 is for cell membrane fusion. ACE2 receptor is present on the ciliated cell of the airway epithelium of human cells; it is called as alveolar type 2 pneumonia site, this receptor also present in the heart, kidney, small intestine, and other tissue. When SARS-COV-2 comes near the by the cells, its S protein (Surface protein) attached to ACE2 ${ }^{(2,7)}$ receptor of the cell for the entrance inside it after that membrane fusion and endocytosis occurs in the cytoplasm and uncoating of the positive RNA genome.

Now polypeptide of viral genome goes under proteolysis process and converted into Non-Structural proteins. Further non-structural proteins become RNA-dependent-RNA polymerase (RdRp) undergoes into RNA synthesis and translation occurs, now these structural proteins assemble like buds on the rough endoplasmic reticulum of Golgi apparatus 
and then transported via vesicles and exocytosis occurs for the viral released to infect other cells.SARS-COV-2 is having a structure of the protein encoded with four types of genes includes Envelope (E), Spike (S), Membrane (M), and Nucleocapsid (N).

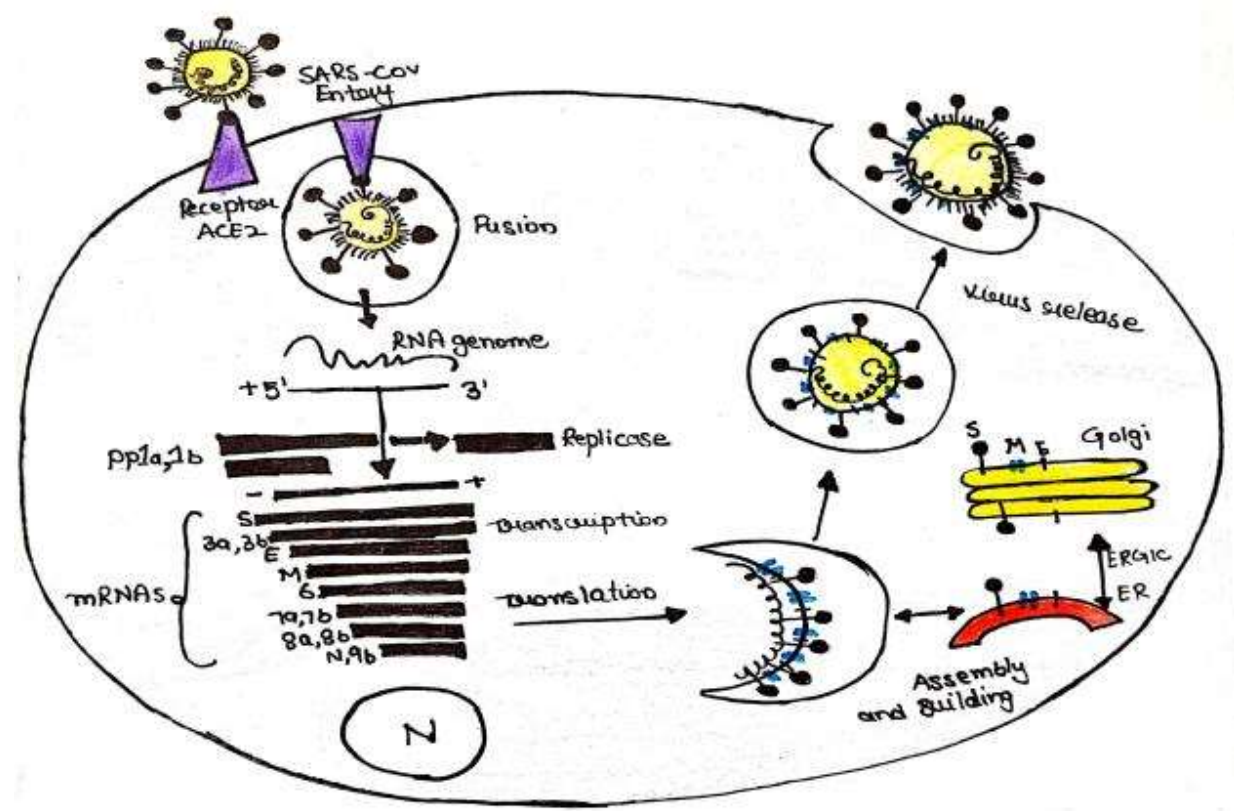

Figure 3: Life cycle of SARS-COV-2 inside the cell
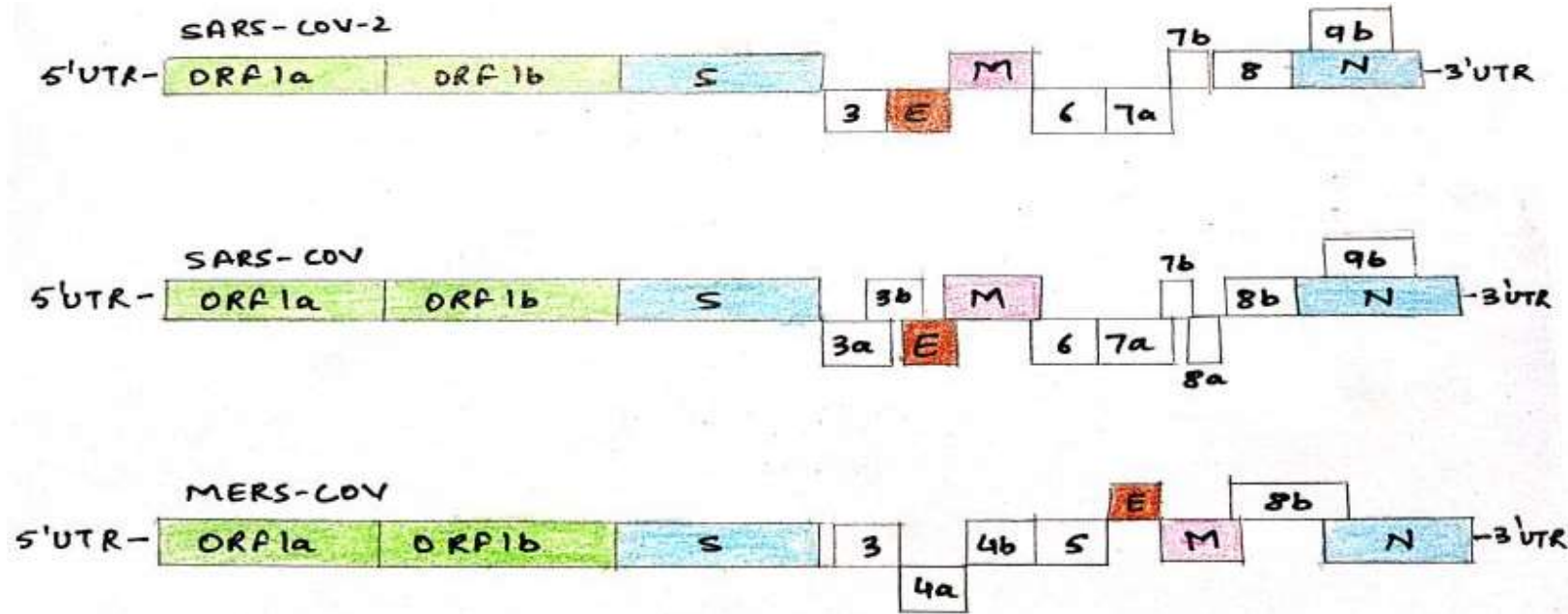

Figure 4: Genomic sequencing of MERS, SARS-COV-1, and SARS-COV-2

The Wuhan coronavirus gene is having spike glycoprotein which can be modified via homologous recombination. Genomic organization of Beta coronaviruses (diagram )of Human Genome consists of 5 ' and untranslated region (5 and UTR ) which encoded with non-structural protein for replication that is open reading frame $1 \mathrm{a} / 1 \mathrm{~b}$, having spike protein structural protein envelop membrane, accessory protein-like orf 3 (SARS-COV-2, MERS-C0V) 3a and 3b (SARS-COV1),4a/4b and 5(MERS-COV), 6a,7a,7b(SARS-COV-1 and SARS-COV-2), 8(SARS-COV-2), 8a(SARS-COV-1),8b(SRASCOV-1 and MERS-COV), 9b(SARS-COV-1ans SARS-COV-2) and the $3^{\prime}$ untranslated region(3' and UTR). The difference between these accessory proteins in all three viruses concludes the variations in the infection. People who are 
having a good immunity system, the virus enters via cell protein receptor that is ACE2 but in case of immunocompromised or patients having comorbidities, cells like macrophages, dendritic, and monocytes are covered with ADE antibodies. When a virus enters in patients it binds to the ADE antibody which is Fc receptors and hence gets enter inside all these types of cells which leads to an increase in both infectivity and virulence of virus which becomes life-threatening. ${ }^{(5)}$

\section{Clinical Manifestation}

The clinical symptoms and evidence are not clear yet but as per reported testimony is ranged from mild to severe, or some cases of death are possible. MERS-COV infection can be asymptomatic but sometimes it also signifies Acute Respiratory Distress Syndrome, pneumonia, septic shock, and multiple organ failure can lead to death. ${ }^{(10)}$ In contrast with SARS-COV1 nearby $75 \%$ of patients having MERS infection had at least one comorbidity who died because of underlined conditions, $42 \%$ of patients were asymptomatic/recovered. Based on collected data of MERS-COV-1 often begins with chills, cough, sore throat, fever, arthralgia, shortness of breath, and myalgia followed by Dyspnea and sudden progression of ammonia within the first week of infection and then frequently receiving organ support and ventilator. Although patients with respiratory illness or who are immunocompromised are symptomatic in the early days, suffer from diarrhoea, chills, fever, and pneumonia. In SRAS-COV-1 and MERS-COV at least one-third of the patient is having gastrointestinal symptoms. ${ }^{(10)}$

In Coronavirus diseases or infection patients who are immunocompromised and having various comorbidities such as diabetes, lung disease, obesity, and Cardiac disease are more prone to this infection. In the case of SARS-COVlinfectious laboratory findings describes chest radiography abnormalities, leukopenia, lymphopenia, thrombocytopenia, high ammonia transferase and increase in lactate dehydrogenase and aspartate ammonia transferase was more compared to MERS infected patients. ${ }^{(10)}$ In the case of COVID-19, the most common symptoms are fatigue, cough, and fever complicated dyspnea, and pneumonia, ${ }^{(3)}$ whereas less common symptoms comprise running nose, headache, diarrhoea, phlegm-producing cough, and hemoptysis. Patients were having mild symptom usually recover after 1 week of infection while patients with severe cases experience growing respiratory failure due to alveolar damage from the virus which may lead to death also. In this infection elderly patient with a pre-existing disease like cirrhosis, coronary heart disease, hypertension, tumour surgery, diabetes, and Parkinson's disease can also result in death. Laboratory findings of SRASCOV-2 show a decrease of lymphocytes and $\mathrm{WBC}$, chest radiography shows new pulmonary infiltrates, there is no improvement in symptoms after the third day of treatment with antibiotics. ${ }^{(3)}$

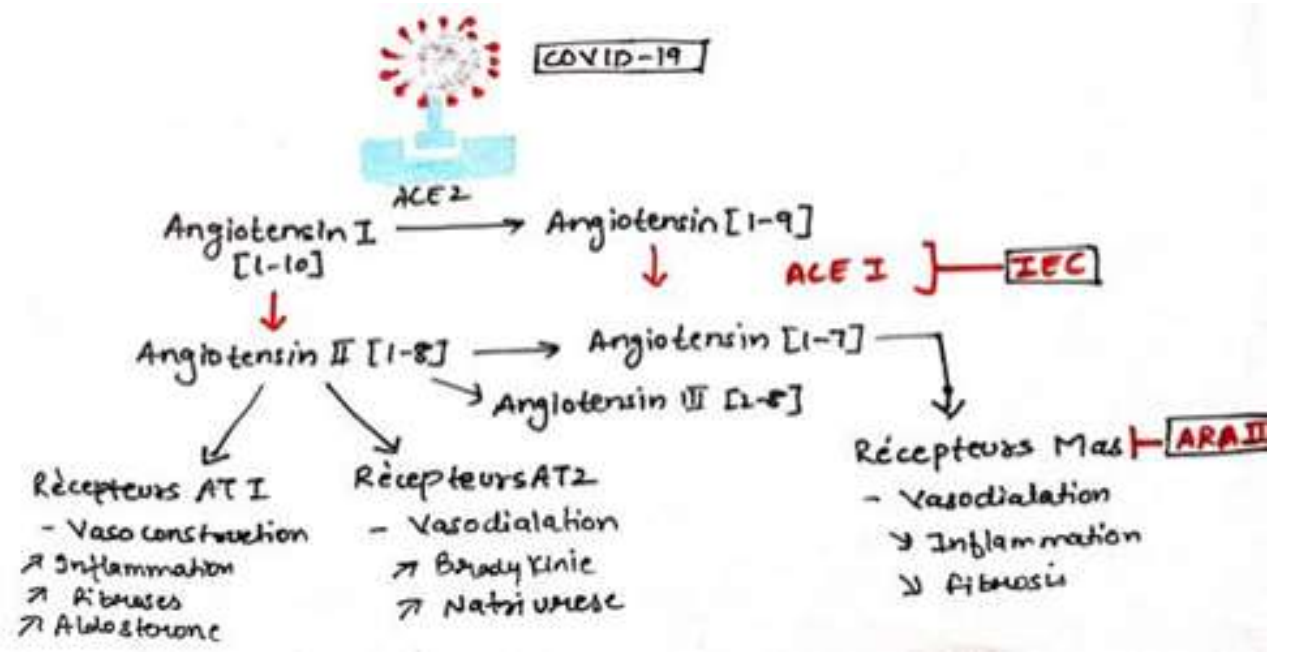

Figure 5: Cell entrance and its effect on the immune system 
Patients who are suspected of this infection have been suggested for real-time Polymerase Chain Reaction (RTPCR) for the diagnosis of the detection of the positive nucleic acid of the virus in different body samples like throat swab, sputum, and lower respiratory secretions. ${ }^{(3)}$ Till the report date, $13.4 \%$ of patients have died and $24 \%$ of patients are recovered hence the fertility rate is increasing day by day of infection. ${ }^{(1)}$

\begin{tabular}{|c|c|c|c|}
\hline Features* & MERS-COV* & SARS-COV-1* & SARS-COV-2* \\
\hline Emergence date & April, 2012 & November,2002 & $\begin{array}{l}\text { December, } \\
2019\end{array}$ \\
\hline Area of emergence & Jordan, Saudi Arabia & Guangdong, China & Wuhan, China \\
\hline Key hosts & Camel & Bats, Raccoon dogs & Bats \\
\hline Number of countries effected & 27 & 29 & 210 \\
\hline Incubation period & 13 days & 2-14 days & 14-21 days \\
\hline \multicolumn{4}{|l|}{ Age group/Sex } \\
\hline Male & 59-64 yrs. & $38-42$ yrs. & $40-60$ yrs. \\
\hline Female & $35-40$ yrs. & 64-68 yrs. & $40-55$ yrs. \\
\hline \multicolumn{4}{|l|}{ Presenting symptoms } \\
\hline Fever & $98 \%$ & $99-100 \%$ & $81-91 \%$ \\
\hline Cough & $83 \%$ & $62-100 \%$ & $48-68 \%$ \\
\hline Diarrhoea & $26 \%$ & $23-70 \%$ & $16 \%$ \\
\hline Nausea and vomiting & $21 \%$ & $20-35 \%$ & $6 \%$ \\
\hline Sore throat & $14 \%$ & $13-25 \%$ & $29 \%$ \\
\hline Headache & $11 \%$ & $20-56 \%$ & $22 \%$ \\
\hline Dyspnea & $22-72 \%$ & $40-42 \%$ & $19-34 \%$ \\
\hline Shortness of breath & $72 \%$ & $40-42 \%$ & $18-20 \%$ \\
\hline Myalgia & $32 \%$ & $45-61 \%$ & $14-16 \%$ \\
\hline Chills & $87 \%$ & $73 \%$ & $11-12 \%$ \\
\hline Hemoptysis & $17 \%$ & $0-1 \%$ & $0.9 \%$ \\
\hline Comorbidities & $76 \%$ & $10-30 \%$ & $35-50 \%$ \\
\hline Diabetes & $10 \%$ & $24 \%$ & $9.2 \%$ \\
\hline Cardiovascular disease & $7.5 \%$ & $10 \%$ & $13.2 \%$ \\
\hline Cancer & $10 \%$ & $6 \%$ & $7.6 \%$ \\
\hline Lung infection & $10 \%$ & $2 \%$ & $8 \%$ \\
\hline Hypertension & $34 \%$ & $19 \%$ & $8.4 \%$ \\
\hline Acute respiratory distress syndrome(ARDS) & $20-30 \%$ & $20 \%$ & $18-30 \%$ \\
\hline Chronic renal disease & $13 \%$ & $2-6 \%$ & $3-5 \%$ \\
\hline Chest Radiography & $92-100 \%$ & $94-100 \%$ & $95-100 \%$ \\
\hline \multicolumn{4}{|l|}{ Laboratory findings } \\
\hline Leukopenia & $14 \%$ & $33 \%$ & $35 \%$ \\
\hline Lymphocytosis & $32 \%$ & $68-85 \%$ & $72 \%$ \\
\hline Thrombocytopenia & $36 \%$ & $40-45 \%$ & $15 \%$ \\
\hline Elevated AST & $14 \%$ & $20-30 \%$ & $38-42 \%$ \\
\hline Elevated ALT & $11 \%$ & $20-30 \%$ & $20-30 \%$ \\
\hline Elevated LDH & $48 \%$ & $50-71 \%$ & $72 \%$ \\
\hline Death rate & $30-40 \%$ & $3.6-15 \%$ & $10-12 \%$ \\
\hline Fatality rate & $41.8 \%$ & $9.6 \%$ & $3-4 \%$ \\
\hline Basic reproduction number & $1 \%$ & $2.3 \%$ & $2.2 \%$ \\
\hline No. of cases & 2260 & 123882 & 7.15Milliion \\
\hline
\end{tabular}

$*(1,2,3,4$, and 10$)$

\section{Familial Cluster Case**}

In January 2020 there was familial cluster of six patients in which patient number 1was asymptomatic (pre-assumed asymptomatic carrier), a 20 years old woman lived in Wuhan and on January 10, 2020, she travelled to Anyang after she reached, she initially met with her two family members (patient 2 and 3) on 10th January 2020. On 13 January 2020, she 
goes along with her five relatives to checkout to another hospitalized relative in Anyang district hospital. Initially, there were no covid-19 cases at the hospital but after she visited the hospital there was the development of disease in her relative who accompanied her. Patient 1 was then isolated and observes critically in the hospital, till 11 February she had no fever, no gastrointestinal, no respiratory symptoms, neither cough nor sour throat was observed. On January 27 and 31, chest City image manifested no remarkable abnormalities, $\mathrm{C}$ reactive protein level, and lymphocyte count were also normal. On 26 January 2020 RT PCR testing was negative, but on 28 January 2020, it showed a positive result, again on 5th and 8th February RT-PCR testing was negative.

From patients 2 to 6 they all develop covid-19. Four Women of age range from 40 to 57 years, none of these patients travel to Wuhan or been in contact with any other infected patient or people who had moved from Wuhan accept patient 1 . From 2 to 5 all developed respiratory syndrome and fever between 23 January and 26 January.

Moreover, they were hospitalized on the same day within the first day RT-PCR of all the patients had come positive. On 17 January 2020, 6th patient develop sore throat and fever and walk to nearby Clinic, and hence, there was no report of covid-19. On 24 January 2020 symptoms of 6 patients were aggravated and get worst over the next few days after that she was hospitalized and on 26 January she got positive for covid-19. Some patient's infection was moderate while patient no. 2 detected with severe pneumonia. All the symptomatic patients had fibrosis, multifocal ground-glass opacities on chest CT. CRP levels were increased and Lymphocytes count was reduced in all the symptomatic patients. This real case shows that the mechanism of asymptomatic Carriers transmission the infection is highly concern because some people are having a good immune system and their body not able to show up the symptoms of infections and hence become the asymptomatic carrier and could able to transfer the infection.

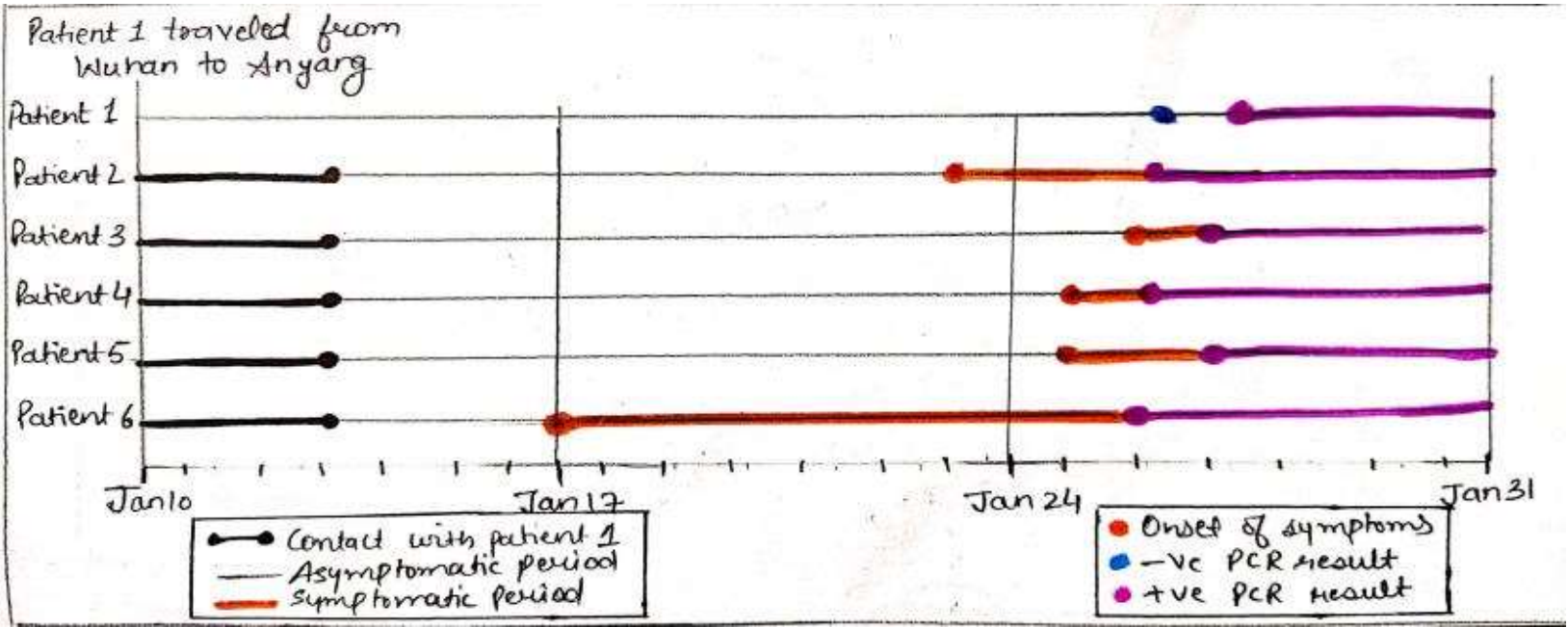

Figure 6: Timeline of Exposure to the Asymptomatic Carrier of the Novel Coronavirus That Causes COVID-19 in a Familial Cluster

$* *(8)$

\section{Diagnosis of SARS-COV-1, MERS-COV, and COVID-19***}

MERS diagnosis can be carried out by Tomography and chest radiography which are compatible with viral pneumonitis and acute respiratory distress syndrome, unilateral or bilateral patchy inflammatory density, ground-glass opacities, with bilateral hilar infiltrates and small pleural effusions in some cases. Upper lobes are generally less affected than lower lobes 
in case of MERS illness with further quick radiographic progressions than in SARS-COV-1. Presence of viral RNA in stool, blood, and urine in some patients but very low viral loads then respiratory tract. Diagnosis from the nasopharyngeal swab from the upper respiratory tract shows less MERS-COV viral loads genome fractions than the lower respiratory tract. Specimen such as bronchoalveolar lavage fluid and tracheal aspirants, who possibly accounts for the incapability of interhuman transmission. Excretion of MERS-COV RNA from the lower respiratory tract can be diagnosed after a week of the illness in most of the patients.

By the detection of viral nucleic acid MERS-COV-1 cases can be confirmed by positive targets on Real-Time PCR on at least two specific genome targets or by a single positive target with Sequencing of the second positive PCR product. In the case of RT PCR test, it includes assays the target on open reading frames ORF1a and ORF1b also the assay target RNA upstream of the E gene. The UPE targeted essay is highly recommended and sensitive for screening and the same within ORF1a assay but less sensitive and useful for information on the case of ORF1b assay. These assays does not shows crossreactivity with other respiratory viruses of human coronavirus to aid the confirmation of MERS-COV to target sites of its genome is suitable for sequencing are RNA dependent RNA polymerase (RdRp) which is present in our ORF1b and N genes. In the case of MERS infection, the case is confirmed by RT-PCR from the sampling of the lower and upper respiratory tract, stool, serum, and urine are also useful to understand the viral load estimation replication, and method of Replication to know the infection control. For the confirmation of infection respiratory samples need to be collected every 2 to 3 days to assay the results after two consecutive negative results. In the first week of illness, the sample is taken, and then for the confirmation of disease by antibody direction paired serum samples need to becollected after 14 to 21 days. $* * *(10)$

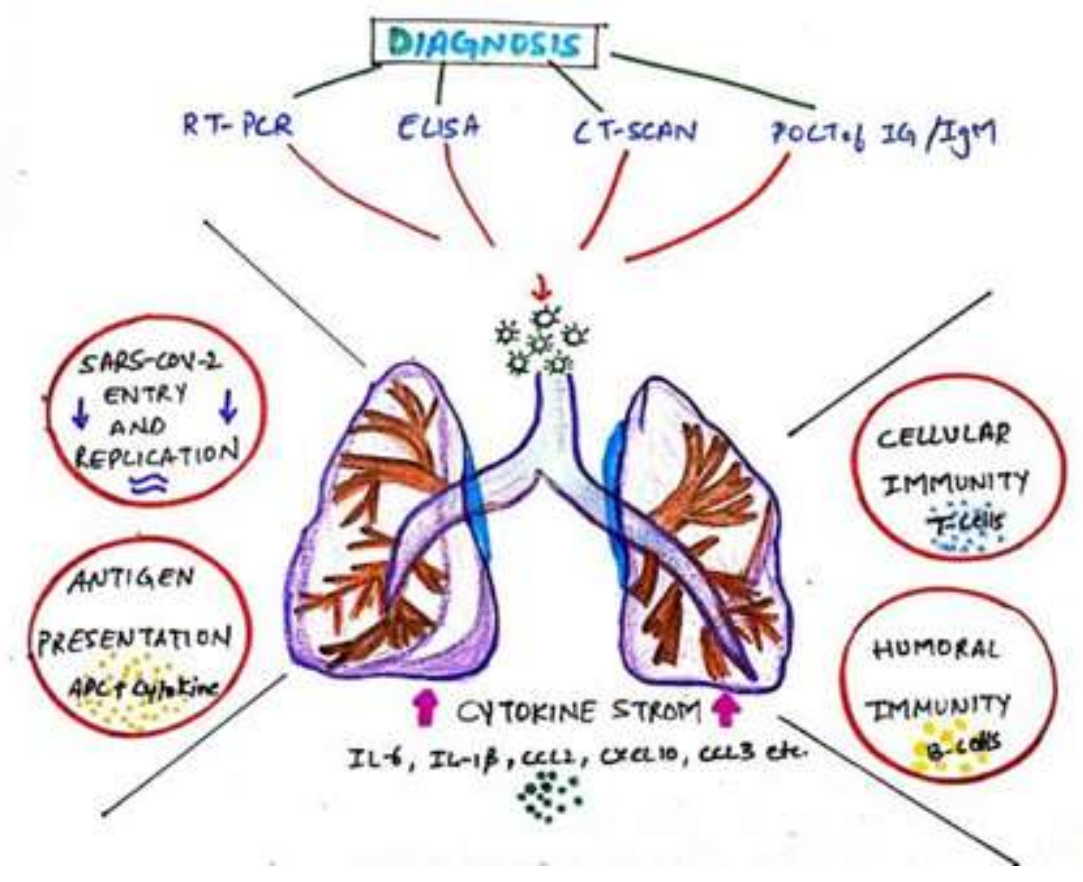

Figure 7: COVID-19 effect on immune cells and its diagnosis

ELISA immunofluorescence assay (positive Sequencing Assay) need to be followed by neutralization assay after the 14 days of the onset of symptoms the sample should be collected and they might be relevant for the recognition of the infection for the cross-reactivity serological test can be useful. Immunohistochemical analysis and in situ hybridization can 
be viable for the detection of covid-19 infection. ${ }^{(6)}$

ELISA: IgM and IgG tests can be combined for efficient clinical diagnosis at multiple stages. EIA-6146-SARSCOV-2 IgG ELISA Kit is used in which covid -19 peptide antigens is used then after the procedure at $450 \mathrm{~nm}$ absorbance reading is taken which confirms the presence of covid-19 antigen in plasma.

RT-PCR: It is also majorly used for the detection of SARS-COV-2 it is having $99 \%$ sensitivity and specificity.

FnCas9 Editor Linked Uniform Detection Assay (FELUDA): This assay is based on a paper strip test that helps to detect the SARS-COV-2 in an hour. It is expected to help and to fulfil an urgent need for rapid testing in India. It works on the principle of Clustered Regularly Interspaced Short Palindromic Repeat Technology (CRISPR). It is a geneediting technology (Disabling gene and fixing technology) that replicates the natural defence mechanisms in the cell to fight with the virus attack. In the case of this technology behaves like a cut and paste mechanism in DNA strands. CRISPR complex finds a target part of the genome with a guide RNA, then it goes along inside the enzyme called CAS enzyme and they connect.

Blood suspected of having coronavirus RNA, lab person add spike in that sample with simple single-stranded DNA or RNA that has a fluorescent reporter system attached to it. If the CRISPR enzyme finds viral RNA, CAS enzyme cuts the viral RNA strands and then attach to it. After that it makes an incision of the molecule holding the reporter which makes it sure florescent in the positive result. This test is six times faster than other diagnostic procedures and it cost less as compared to RT-PCR.

\section{Predictions}

Combination of 6 medicines with the appropriate and required amount which can be able to inhibit the S protein binding that will show immunosuppressant activity, anti-inflammation, helps to inhibit the replication of viral RNA with the human genome. We can also use the combination of 2 or 3 medicines like in various ways like of capsule in a capsule having powdered, gel or granular forms of any another chemical or medicinal substrate as a nutraceutical product with natural additives like as Vitamin D is also known profound beneficial for the COVID-19 as it helps to boost immunity which could be able to maintain the body.

A combination of Camostat mesylate and Arbidol/ Chloroquine Hydroxychloroquine can be given in some required amount to first inhibit TMPRSS2 which probably prevents the entry of viruses in the cell through S spike protein and then to inhibits the endocytosis to stops the further mechanism respectively. Lopinavir/Darunavir, Ribavirin/Remdesivir/Favipiravir, Tocilizumab/Sarilumab all are some other drugs that can be used effectively in the treatment of coronavirus.

Tocilizumab or Sarilumab medicines help to inactivate the IL-6 receptor by binding to it and hence to inhibit the signalling. Lopinavir or Darunavir is further can have a medicinal effect in the inhibition of 3-chymotrypsin like protease to stop the polypeptides conversion into non-structural proteins with the proteolysis process. Therefore, no other process can happen and consequently no infection in the body. Now, same with other medications the Ribavirin, Remdesivir, and Favipiravir is also having a satisfactory effect in the inhibition of viral RdRp so that viral RNA could not be able to synthesis the DNA or cRNA strand with the human genome.

We can prepare a vaccine in which the chemical substrate work and able to make a shield around the virus. As the 
virus enters that substrate becomes active and covers that virus genome so that it could not able attach to cells and infect further.

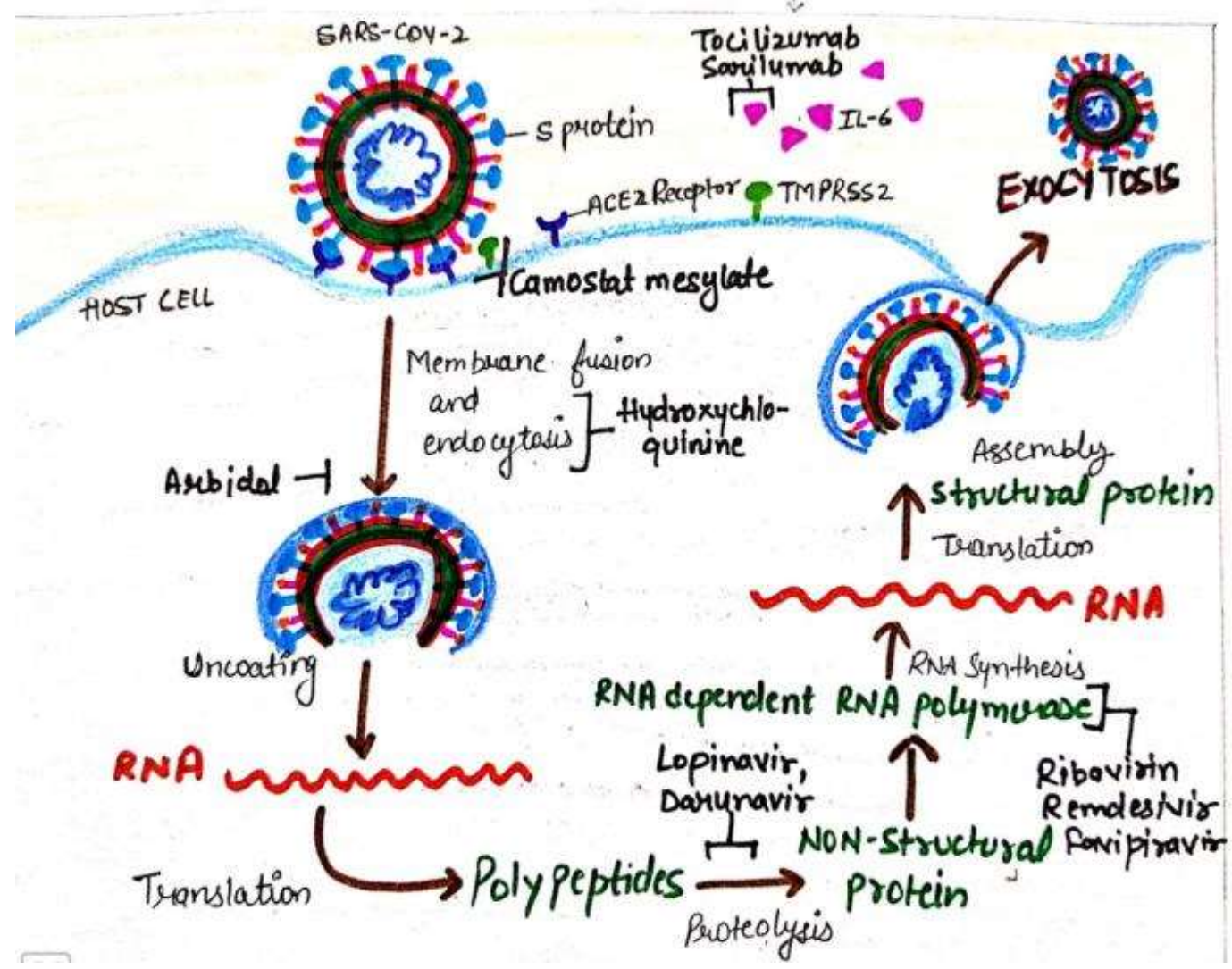

Figure 8: Effect of Medications on the Sars-covid-2 infection inside the cell.

\section{Treatment}

Hydrochloroquinine is considered as the need and required medication or drug for the treatment of covid infection. The medicinal course of this medicament treatment is for 14 days at 3-days of interval. For Adults, 1 Tablet is of 200mg and 2 Tablets are given at a time and twice in a day (Morning and Evening) with $500 \mathrm{ml}$ of Normal Saline. If a person is having fever then Paracetamol in IV Stat is given and for the person with congestion, Asthaline nebulizer is given for the relaxation of the respiratory tract. Other combinations or a blend of drugs like HIV, Hepatitis, and Swine flu are given by doctors but its quantity depends upon the patient's history, condition, and symptoms.

In case of children age from $10-13$ yrs. or above 50mg 2 tablets if given the same as the normal procedure twice a day on 3-days of interval for 14 days. For infants, Hydrochloroquinine syrup of $222.5 \mathrm{mg}$ is given.

Some patients are allergic to tablets so in that case IV Stat of HCQ (liquid or powder) in high Normal Saline. Moreover, the dosage is different for every separate individual likewise some patients are having Diabetes, CVD problems, and pneumonia so in that case the dosage and treatment and dosage would be modified. In case of Blood pressure, HCQ acts fast and increases the blood flow rate thus in that case HCQ is given whether in low quantity or the liquid form in Normal Saline. 


\section{Control and Preventions $* * * *$}

WHO, the US centers for disease control and prevention recommended that infection prevention and control measure includes contact precautions in which it comprises wearing gloves and gowns while coming in contact with infected patients and droplets precautions incorporate to wear a protective mask. For health care workers eye protection is a must when they come in contact with patients. WHO also guides to use personal protective equipment for instance gloves; face shield (eye protection), gowns, and respiratory protection, that is, a disposable N95 filtering facepiece respirator. Covid-19 patients should be assigned to negative pressure rooms or rooms having exhausted is filtered by the high-efficiency particulate air filter. For air-bone precaution, aerosol-generating procedures should be applied in the rooms.

Foods that we are customizing from the market need to be washed and cooked properly. Good personal hygiene which includes regular hand washing avoids touching nose, eye, and mouth with hands and avoids contact with covid patients. Social distancing and lockdown announced by the Governments at the national level in some countries. All the parts of countries actively suspended activities such as markets cinema, sports events, schools, universities, offices, airports, railways, roadways, and other crowded places are postponed. Various quarters or compartments, institutions, and hospitals are setups as quarantine isolation wards.

Emergency Material Support: Governmental authorities restored production and expanded production capacities, supported local enterprises and e-commerce platforms, and provide medical facilities and basic needs which are to be fulfilled. ${ }^{* * * *(1)}$

\section{CONCLUSIONS}

MERS and SARS-COV-1 were not much pandemics than SARS-COV-2. In 2019 December, this virus came and infected people that showed symptoms similar to ARDS and swine flu but after 1-2 months its shows variations and sometimes similar like pre-existing diseases only. The outbreak of COVID-19 globally affects many countries and people of almost every country and people of every age group. Patients with various comorbidities having difficulty in cure from this infection and can also lead to death but sometimes because of good immune health person can heal. Transmission can be direct or indirect and some patients may be symptomatic or asymptomatic. SARCS-COV-2 shows variations in its genome sequences and hence creating difficulty to discover its acceptable vaccination. Epidemiology shows the fatality and mortality rate of MERS, SARS-COV-1, and SARS-COV-2 infection. Pathogenesis of pre-existing viruses and COVID-19 is contrasting in some way but all viruses from this family infect the respiratory tract on the initial stage then infect other body organs. Differences between genomic sequences of the three viruses helping to discrete from each other. Clinical manifestation shows the rate of symptoms and laboratory findings. The difference between these viruses shows the variations in their infection, symptoms, death and fatality rate, and several cases, etc. The familial cluster case shows that the mode of transmission can be from asymptomatic patients. Diagnosis is done by various methods majorly with RT-PCR, ELISA, and FELUDA which are very sensitive and specific for its testing. Prediction for the treatment and the medications that are already in use shows that it might be difficult to discover drugs and vaccines for the virus but not unattainable. Control and preventive measures need to be followed to control and to improve its outbreak in all over the world.

\section{REFERENCES}

1. Report of the WHO-China Joint Mission on Coronavirus Disease 2019 (COVID-19).

2. N. Petrosillo, G. Viceconte, O. Ergonul, G. Ippolito , E. Petersen COVID-19, SARS and MERS: are they closely related?, 
Clinical Microbiology and Infection, https://doi.org/10.1016/j.cmi.2020.03.026

3. Epidemiology, causes, clinical manifestation and diagnosis, prevention and control of coronavirus disease (COVID-19) during the early outbreak period: a scoping review

4. Singh, Brijesh P. "Modeling and Forecasting Novel Corona Cases in India Using Truncated Information: A Mathematical Approach." International Journal of Applied Mathematics \& Statistical Sciences 9.4 (2020): 13-24.

5. Sasmita Poudel Adhikari, Sha Meng, Yu-Ju Wu, Yu-Ping Mao, Rui-Xue Ye, Qing-Zhi Wang, Chang Sun, Sean Sylvia, Scott Rozelle, Hein Raat and Huan Zhou https://doi.org/10.1186/s40249-020-00646-x

6. Severe acute respiratory syndrome vs. the Middle East respiratory syndrome David S. Hui, Ziad A. Memish, and Alimuddin Zumla DOI:10.1097/MCP.0000000000000046

7. COVID-19 infection: origin, transmission, and characteristics of human coronaviruses Muhammad Adnan Shereen, Suliman Khan, Abeer Kazmi, Nadia Bashir, Rabeea Siddique PII: S2090-1232(20)30054-0, https://doi.org/10.1016/j.jare.2020.03.005

8. The Severe Acute Respiratory Syndrome Joseph S.M. Peiris, M.D., D.Phil., Kwok Y. Yuen, M.D., Albert D.M.E. Osterhaus, Ph.D., and Klaus Stöhr, Ph.D., N Engl J Med 2003;349:2431-41.

9. NGUYEN, DONGTHI THAO, and KIEUTHI THU CHUNG. "NEW TRENDS IN TECHNOLOGY APPLICATION IN EDUCATION AND CAPACITIES OF UNIVERSITIES LECTURERS DURING THE COVID-19 PANDEMIC." International Journal of Mechanical and Production Engineering Research and Development (IJMPERD) 10.3, Jun 2020, 1709-1714

10. Coronavirus infections: Epidemiological, clinical and immunological features and hypotheses Didier Raoult, Alimuddin Zumla, Franco Locatelli, Giuseppe Ippolito and Guido Kroemer, doi: 10.15698/cst2020.04.216

11. Research letter, Yan Bai, MD, Lingsheng Yao,MD, TaoWei, MD, Fei Tian, MD, Dong-Yan Jin, PhD, Lijuan Chen, PhD, MeiyunWang,MD, PhD February 21, 2020. doi:10.1001/jama.2020.2565

12. A pandemic risk assessment of Middle East respiratory syndrome coronavirus (MERS-CoV) in Saudi Arabia Saleh A. Eifan, Islam Nour, Atif Hanif, Abdelrahman M.M. Zamzam, Sameera Mohammed AlJohani PII: S1319-562X(17)30150-X, http://dx.doi.org/10.1016/j.sjbs.2017.06.001

13. Middle East respiratory syndrome Alimuddin Zumla, David S Hui, Stanley Perlman http://dx.doi.org/10.1016/S0140$\underline{6736(15) 60454-8}$

14. Coronavirus Infections-More Than Just the Common Cold Catharine I. Paules, MD, HilaryD. Marston,MD, MPH, Anthony S. Fauci,MD doi:10.1001/jama.2020.0757

15. From SARS coronavirus to novel animal and human coronaviruses Kelvin K. W. , Ivan F. N. Hung, Jasper F. W. Chan, KwokYung Yuen, J Thorac Dis 2013;5(S2):S103-S108. doi: 10.3978/j.issn.2072-1439.2013.06.02 
